\title{
Proving Positive Almost-Sure Termination
}

\author{
Olivier Bournez, Florent Garnier \\ LORIA/INRIA, 615 Rue du Jardin Botanique \\ 54602 Villers lès Nancy Cedex, France
}

\begin{abstract}
In order to extend the modeling capabilities of rewriting systems, it is rather natural to consider that the firing of rules can be subject to some probabilistic laws. Considering rewrite rules subject to probabilities leads to numerous questions about the underlying notions and results.

We focus here on the problem of termination of a set of probabilistic rewrite rules. A probabilistic rewrite system is said almost surely terminating if the probability that a derivation leads to a normal form is one. Such a system is said positively almost surely terminating if furthermore the mean length of a derivation is finite. We provide several results and techniques in order to prove positive almost sure termination of a given set of probabilistic rewrite rules. All these techniques subsume classical ones for non-probabilistic systems.
\end{abstract}

\section{Introduction}

Since 30 years, term rewriting has shown to be a very powerful tool in several contexts where efficient methods for reasoning with equations are required $[1,13]$. In the last decade, term rewriting has also shown to provide a very elegant framework for specifying concurrency models and deduction systems [16,17].

When specifying probabilistic systems, it is rather natural to consider that the firing of a rewrite rule can be subject to some probabilistic rules. For that purpose, we proposed in [4] to add basic probabilistic strategies to rule based languages. The idea of adding probabilities to rewrite rules has also been explored in [9] in the context of probabilistic constraint handling rules, or in [18]. The idea of adding probabilities to high level models of reactive systems has also been explored for models like Petri Nets [2,22], automata based models [6,26], or process algebra [11].

Considering rewrite rules subject to probabilities leads to numerous questions about the underlying notions and results. In [4], we introduced probabilistic abstract reduction systems, and we introduced notions like almost-sure termination or probabilistic confluence, with relations between all these notions. In [3], we proved that, unlike what happens for classical rewriting logic, there is no hope to build a sound and complete proof system with probabilities in the general case. We however proposed a rather natural notion of rewriting logic which is sound and complete when proof terms are explicit [3].

This paper is a contribution devoted to a next step: understand and provide proof techniques for proving termination of a set of probabilistic rewrite rules. 
As in [4], we propose to call a deterministic probabilistic rewrite system almost surely terminating if the probability that a term leads to a normal form is one. However, unlike in [4], we also allow non-deterministic systems. A nondeterministic probabilistic rewrite system is said almost surely terminating if the probability that a term leads to a normal form is one whatever the reduction strategy is.

The idea of mixing probabilities with non-determinism in several other high level models for reactive systems has quite extensively been discussed in literature. To solve semantical problems, discussed for example in [19] or [12], several approaches exist. One of them, called the generative approach [25], consists in ruling out non-determinism by means of a probability distribution that assigns a probability to each possible action. The reactive approach [25] consists in allowing both non-deterministic and probabilistic transitions. The present nondeterminism is solved using the notion of schedulers [26]. Note that there exist intermediate approaches such that stratified approaches [25] or simple or fully probabilistic transition systems [24] that are variants or combinations of these two approaches. Our approach is close to the reactive approach, and what we call a probabilistic abstract reduction system is also called a Markov decision process in other contexts [20].

Termination is a desirable interesting notion. However, in the probabilistic context, we think we should distinguish "reasonable" termination from general termination.

Indeed, consider a system like a symmetric random walk on the set $\mathbb{Z}^{k}$ of integers. For $k=1$ or 2 , it visits almost surely all the points $[7,5]$. Hence, whatever the current position is, if one wants to go to the origin, a strategy is to evolve like a symmetric random walk and stop at the origin. However, even if one is almost sure to reach the origin, the expected time before reaching the origin is infinite $[5,7]$.

Coming back to termination, the point is that in an almost surely terminating system, with probability one a term leads to a normal form, but if the mean number of a derivation is infinite then this information is rather useless.

Hence, we believe that the following notion is more interesting: a system will be said positively almost surely terminating if the mean length of a derivation is finite. After formally introducing all these notions, we will see that positive almost sure termination implies almost sure termination. The rest of this paper is then devoted to proof techniques that can be used to prove positive almost sure termination.

In particular, in the classical non-probabilistic case, a simple and often used criteria for proving termination consists in embedding the underlying abstract reduction system into the set of natural integers, in such a way that each transition corresponds to a decreasing transition. This technique is sound in the general case, and is complete for finitely branching systems [1].

We show that this technique has an equivalent for probabilistic abstract reduction systems: we prove that a probabilistic abstract reduction system is positively almost sure terminating if it can be embedded into the set of non-negative 
reals in such a way that each transition corresponds to a decreasing transition in mean. The technique is proved sound in the general case, and complete for finitely branching systems.

Benefiting from the possibility of considering non-deterministic probabilistic abstract reduction systems, we then define probabilistic rewrite systems. The idea is to allow in right hand sides of probabilistic rules a distribution on classical right hand sides of classical rewrite rules. The proposed notions are intended to subsume classical rewrite systems. In that spirit, they seem rather natural (at least for the rewrite community) and probabilistic rewrite systems provide an alternative to the numerous probabilistic high level formalisms for specifying reactive systems.

We then discuss the equivalent of the classical result that says that a rewrite system is terminating iff there is a reduction order monotone on each rewrite rule.

The paper is organized as follows: in Section 2, we recall classical nonprobabilistic theory. Sections 3 and 4 recall basic probability and Markov chain theory, and Foster's theorem respectively. Section 5 introduces probabilistic abstract reduction systems. Section 6 defines positive almost sure termination. Section 7 provides techniques for proving positive almost sure termination of a probabilistic abstract reduction system. Probabilistic rewrite systems are introduced in Section 8. Techniques for proving their positive almost sure termination are discussed in Section 9.

\section{Termination and Abstract Reduction Systems}

We first come back to the classical setting: see for example [1,13]. An abstract reduction system $(A R S)$ is $\mathcal{A}=(A, \rightarrow)$ consisting of a set $A$ and a binary relation $\rightarrow \subset A \times A$ on $A$. A derivation is a finite, or infinite sequence $\pi=\pi_{0} \rightarrow \pi_{1} \cdots \rightarrow$ $\pi_{n}$ with $\left(\pi_{i}, \pi_{i+1}\right) \in \rightarrow$ for all $i$. An abstract reduction system is said terminating iff there is no infinite chain $a_{0} \rightarrow a_{1} \rightarrow \cdots$.

As said in [1], the most basic method for proving termination of some $\mathcal{A}=$ $(A, \rightarrow)$ is to embed it into another abstract reduction system $\mathcal{B}=(B,>)$ which is known to terminate. This require a monotone mapping $V: A \rightarrow B$, where monotone means that $x \rightarrow x^{\prime}$ implies $V(x)>V\left(x^{\prime}\right)$. Now $\rightarrow$ terminates because an infinite chain

$$
a_{0} \rightarrow a_{1} \rightarrow \cdots
$$

would induce an infinite chain

$$
V\left(x_{0}\right)>V\left(x_{1}\right)>\ldots
$$

The most popular choice for termination proofs is an embedding into $(\mathbb{N},>)$. Its popularity comes partly from the following easy completeness result [1].

Proposition 1. A finitely branching abstract reduction system terminates if and only if there is a monotone embedding into $(\mathbb{N},>)$. 
As in [1], observe that the technique is sound in the general case, but complete only for finitely branching systems. Indeed, the system with $A=\mathbb{N}^{2}$ and $\rightarrow$ defined by $(i+1, j) \rightarrow(i, k),(i, j+1) \rightarrow(i, j)$, for all $i, j, k$, is terminating, whereas there is no monotone embedding from $\left(\mathbb{N}^{2}, \rightarrow\right)$ to $(\mathbb{N},>)[1]$.

\section{Stochastic Sequences and Markov Chains}

Let us first come back to school [10,7,21]: a $\sigma$-algebra on a set $\Omega$ is a set of subsets of $\Omega$ which contains the empty-set, and is stable by countable union and complementation. In particular, the set of subsets is a natural $\sigma$-algebra for any countable set. A measurable space $(\Omega, \sigma)$ is a set with a $\sigma$-algebra on it. A probability is a function $P$ from a $\sigma$-algebra to $[0,1]$, which is countably additive, and such that $P(\Omega)=1$. A triplet $(\Omega, \sigma, P)$ is called a probability space.

If $(\Omega, \sigma)$ and $\left(\Omega^{\prime}, \sigma^{\prime}\right)$ are measurable spaces, a function $f: \Omega \rightarrow \Omega^{\prime}$ is measurable if for all $W$ in $\sigma^{\prime}, f^{-1}(W) \in \sigma$. A random variable is a measurable function on some probability space. The mean of a random variable $V$ taking values in the set $\mathbb{N}$ of integers is $E[V]=\sum_{i} i P(V=i)$. This value is always defined, even if it can be finite or infinite. Observe that such a random variable always satisfy the so-called telescope formula $E[X]=\sum_{n=0}^{\infty} P(X>n)$ [5]. For a random variable $V$ taking values in $\mathbb{N} \cup\{+\infty\}$, the mean $E[V]$ can still always be defined: practically, it is infinite if $P(V=+\infty)>0$ and equal to $E[V]=\sum_{i} i P(V=i)$ (which may still be infinite) otherwise.

Given $A, B \in \sigma$, when $P(B)>0$, the conditional probability of $A$ given $B$ is by definition $P(A \mid B)=P(A \cap B) / P(B)$. The mean of random variable $V: \Omega \rightarrow \mathbb{N}$ conditioned by $B$ is defined by $E[V \mid B]=\sum_{i} i P(V=i \mid B)$.

A stochastic sequence on a set $A$ is a family $\left(X_{i}\right)_{i \in \mathbb{N}}$, of random variables defined on some fixed probability space $(\Omega, \sigma, P)$ with values on $A$. It is said to be Markovian if its conditional distribution function satisfies the so-called Markov property, that is for all $n$ and $s \in A$,

$$
P\left(X_{n}=s \mid X_{0}=\pi_{0}, X_{1}=\pi_{1}, \ldots, X_{n-1}=\pi_{n-1}\right)=P\left(X_{n}=s \mid X_{n-1}=\pi_{n-1}\right),
$$

and homogeneous if furthermore this probability is independent of $n$.

The matrix $\left(p_{s, t}^{i}\right)=\left(P\left(X_{i+1}=t \mid X_{i}=s\right)\right)$ is what is called a stochastic matrix (even when $A$ is an infinite set) [5]. It has the nice property that columns sum to 1 .

Giving a Markov Chain is of course equivalent to giving the sequence of its stochastic matrices. Given a Homogeneous Markov Chain corresponds to giving a unique stochastic matrix (at any rank, the matrix is the same).

\section{Foster's theorem}

We are searching criteria in the spirit of Proposition 1. For that purpose, we now state the following result, that can be attributed to Foster [8]. It has strong connections with Martingale theory and can be seen as a consequence of very general 
results of (super) Martingale theory. However, it can be proved independently as in [5].

Theorem 1 (Foster's Theorem). Given a homogeneous Markov chain over a countable space $A$ whose matrix is $P=\left(p_{t, s}\right)$, if there exists a measurable subset $C \subset A$, and some function $V: A \rightarrow \mathbb{R}$, with $\inf _{i \in A} V(i)>-\infty$ and such that the mean drift defined by $\Delta V(i)=\sum_{k \in A} p_{i, k} V(k)-V(i)$ satisfies for some $\epsilon>0$ $\Delta V(i) \leq-\epsilon$ for all $i \notin C$, then almost surely one reaches $C$.

Furthermore, the mean time to reach $C$ from $i$ is finite and less than $V(i) / \epsilon$.

Notice that the technique of using Foster's theorem in order to prove convergence to some set $C$ has similarities with techniques used in self-stabilization as in $[23,15]$.

\section{Probabilistic Abstract Reduction Systems}

We are now ready to define probabilistic abstract reduction systems (PARS). We define PARS in a slightly modified way to [4]. The main motivation is that we want to allow non-deterministic systems.

In the same way that abstract reduction systems are also called transition systems in other contexts, PARS can be considered as Markov Decision Processes [20]. The only point is that, compared to usual definitions of Markov decision processes, we explicitly allow states to be terminal, and that we do not label transitions by actions.

The idea is that a PARS is given by some set $A$, and a relation that relate states to distributions on their successors.

Definition 1 (PARS). Given some denumerable set $S$, we note Dist $(S)$ for the set of probability distributions on $S: \mu \in \operatorname{Dist}(S)$ is a function $S \rightarrow[0,1]$ that satisfies $\sum_{i \in S} \mu(i)=1$.

A probabilistic abstract reduction system $(P A R S)$ is a pair $\mathcal{A}=(A, \rightarrow)$ consisting of a countable set $A$ and a relation $\rightarrow \subset A \times \operatorname{Dist}(A)$.

A PARS is said deterministic if, for all a, there is at most one $\mu$ with $a \rightarrow \mu$.

$A$ state $a \in A$ with no $\mu$ such that $a \rightarrow \mu$ is said terminal.

We now need to explain how such systems evolve: a history (of length $n+1$ ) is a finite sequence $a_{0} a_{1} \cdots a_{n}$ of elements of the state space $A$. It is non-terminal if $a_{n}$ is. A policy $\phi$, that can also be called a strategy, is a function that maps non-terminal histories to distributions in such a way that $\phi\left(a_{0} a_{1} \cdots a_{n}\right)=\mu$ is always one (of the possibly many) distribution $\mu$ with $a_{n} \rightarrow \mu$. A history is said realizable, if for all $i<n$, if $\mu_{i}$ denotes $\phi\left(a_{0} a_{1} \cdots a_{i}\right)$, one has $\mu_{i}\left(a_{i+1}\right)>0$.

A derivation of $\mathcal{A}$ is then a stochastic sequence where the non-deterministic choices are given by some policy $\phi$, and the probabilistic choices are governed by the corresponding distributions.

Formally: 
Definition 2 (Derivations). A derivation $\pi$ of $\mathcal{A}$ over policy $\phi$ is a stochastic sequence $\pi=\left(\pi_{i}\right)_{i \in \mathbb{N}}$ on $A \cup\{\perp\}$ such that for all $n$,

$$
\begin{gathered}
P\left(\pi_{n+1}=\perp \mid \pi_{n}=\perp\right)=1, \\
P\left(\pi_{n+1}=\perp \mid \pi_{n}=s\right)=1 \text { if } s \in A \text { is terminal, } \\
P\left(\pi_{n+1}=\perp \mid \pi_{n}=s\right)=0 \text { if } s \in A \text { is non-terminal, }
\end{gathered}
$$

and for all $t \in A$.

$$
P\left(\pi_{n+1}=t \mid \pi_{n}=a_{n}, \pi_{n-1}=a_{n-1}, \ldots, \pi_{0}=a_{0}\right)=\mu(t)
$$

whenever $a_{0} a_{1} \cdots a_{n}$ is a realizable non-terminal history and $\mu=\phi\left(a_{0} a_{1} \cdots a_{n}\right)$.

Several observations are in order.

Remark 1. Deterministic probabilistic abstract reduction systems correspond to probabilistic abstract reduction systems considered in [4].

Remark 2. The derivations are homogeneous and Markovian when the policy $\phi$ is Markovian, i.e. when the value of $\phi\left(a_{0} a_{1} \ldots a_{n}\right)$ depends only on the value of $a_{n}$. In particular, this holds for deterministic systems.

\section{Termination of a Probabilistic Abstract Reduction System}

If a derivation is such that $\pi_{n}=\perp$ for some $n$, then $\pi_{n^{\prime}}=\perp$ almost surely for all $n^{\prime} \geq n$. Such a derivation is said to be terminating. In other words, a non-terminating derivation is such that $\pi_{n} \in A\left(\pi_{n} \neq \perp\right)$ for all $n$.

Definition 3 (Almost Sure Termination). A PARS $\mathcal{A}=(A, \rightarrow)$ will be said almost surely (a.s) terminating iff for any policy $\phi$, the probability that a derivation $\pi=\left(\pi_{i}\right)_{i \in \mathbb{N}}$ under policy $\phi$ terminates is 1: i.e. for all $\phi, P\left(\exists n \mid \pi_{n}=\right.$ $\perp)=1$.

This can be restated as follows: given some policy $\phi$, and some state $a$, consider the random variable $\tau[a, \phi]$ associated to a derivation $\pi$ with $\pi_{0}=a$, taking values in $\mathbb{N} \cup\{+\infty\}$, defined as $+\infty$ if $\pi_{n} \neq \perp$ for all $n$, and defined as $\tau[a, \phi]=\min \left\{n \mid \pi_{n}=\perp\right\}$ otherwise. Of course, $\tau[a, \phi]$ corresponds to the number of derivations from $a$ under strategy $\phi$ before termination. $\tau[a, \phi]$ is easily proved to be a stopping time for all $\phi$ and $a$.

Previous definitions can then be stated as follows:

Proposition 2. A PARS $\mathcal{A}$ is almost surely terminating iff for all strategies $\phi$ and all states $a, P(\tau[a, \phi]=+\infty)=0$. 
As discussed in the introduction, this notion of termination is too weak. Even if $P(\tau[a, \phi]=\infty)=0$, it might happen that the mean time before termination

$$
T[a, \phi]=E[\tau(a, \phi)]
$$

is not finite, and one may expect never to reach a terminal state.

That is why, we suggest to introduce the notion of positive almost sure termination. Note that the choice of the name "positive" is inspired by the distinction between positive recurrence and null recurrence in Markov chains theory [5].

Definition 4 (Positive Almost Sure Termination). A PARS $\mathcal{A}=(A, \rightarrow)$ will be said positively almost surely (+a.s.) terminating if for all policies $\phi$, for all states $a \in A, T[a, \phi]$ is finite.

By the discussion in Section 3 on random variables taking values in $\mathbb{N} \cup\{+\infty\}$, we know that if $P(\tau(a, \phi)=\infty)>0$ then necessarily $E[\tau(a, \phi)]$ is infinite. That means:

Proposition 3. A positively almost surely terminating PARS is almost surely terminating.

Remark 3. The previous notions subsume classical ones. As one may expect, non-probabilistic systems are special cases of probabilistic systems: an abstract reduction system is a probabilistic abstract reduction system where all the distributions are Dirac distributions. I.e. all the distributions $\mu$ have value 1 on a single point, and value 0 everywhere else. Strategies for abstract reduction systems do indeed correspond to strategies for corresponding probabilistic abstract reduction systems. Terminating derivations for abstract reduction systems do indeed correspond to terminating derivations for corresponding probabilistic abstract reduction systems. An abstract reduction system is terminating iff the corresponding probabilistic abstract reduction system is ((positively) almost surely) terminating. Note that positive almost sure termination corresponds to almost sure termination and to termination for those systems.

\section{Proving positive almost sure termination}

We are now going to discuss techniques for proving positive almost sure termination of a probabilistic abstract reduction system. We propose a technique that subsumes the technique of Proposition 1.

One must understand that it is not at all a coincidence, but more or less unavoidable: a deep consequence of remark 3 is that any technique for proving positive almost surely termination of probabilistic abstract reduction systems must also work for abstract reduction systems, and hence necessarily subsumes a technique for non-probabilistic abstract reduction systems.

First, we prove soundness of our technique 
Theorem 2 (Soundness). A PARS $\mathcal{A}=(A, \rightarrow)$ is +a.s. terminating if there exist some function $V: A \rightarrow \mathbb{R}$, with $\inf _{i \in A} V(i)>-\infty$, and some $\epsilon>0$, such that, for all states $a \in A$, for all $\mu$ with $a \rightarrow \mu$, the drift in a according to $\mu$ defined by

$$
\Delta_{\mu} V(a)=\sum_{i} \mu(i) V(i)-V(a)
$$

satisfies

$$
\Delta_{\mu} V(a) \leq-\epsilon .
$$

Proof. We would like to use Theorem 1. However, we can not work directly on the PARS, since even if we fix a strategy, a PARS is not necessarily an homogeneous Markov chain (the fixed policy can be non-Markovian).

The solution is to fix a policy $\phi$ and to work on an homogeneous Markov chain $\mathcal{M}_{\phi}$ defined on another state space: the state space of $\mathcal{M}_{\phi}$ is defined as the set of all realizable histories of $\mathcal{A}$.

The matrix of Markov chain $\mathcal{M}_{\phi}$ is then defined such that

- for all $t, p_{h, h t}=\mu(t)$ where $\mu=\phi(h)$ if $h=a_{0} a_{1} \cdots a_{n}$ is a realizable non-terminal history, where $h t$ stands for history $a_{0} a_{1} \cdots a_{n} t$,

$-p_{h, h}=1$ if $h$ is a realizable terminal history,

- and every other entry of the matrix is 0 .

By construction, $\mathcal{M}_{\phi}$ is an homogeneous Markov chain. Now clearly, a trajectory of PARS $\mathcal{A}$ starting from $a$ reaches a terminal state under policy $\phi$ iff the corresponding trajectory of $\mathcal{M}_{\phi}$ of same length starting from a leads to a terminal history. Furthermore, the probabilities of corresponding derivations are preserved.

Consider now function $W: S_{\phi} \rightarrow \mathbb{R}$ defined by

$$
W\left(a_{0} a_{1} \cdots a_{n}\right)=V\left(a_{n}\right)
$$

for all realizable histories $a_{0} a_{1} \cdots a_{n}$.

We have

$$
\Delta W(h)=\Delta_{\mu} V(h) \leq-\epsilon
$$

for any non-terminal realizable history $h$, where $\mu=\phi(h)$.

We can then apply Theorem 1 on $\mathcal{M}_{\phi}$, with $C$ equal to the set of terminal realizable histories to conclude that the derivations starting from $a$ in $\mathcal{M}_{\phi}$ reach terminal realizable histories in a time whose mean is less than $W(a) / \epsilon=V(a) / \epsilon$.

Hence, all the derivations starting from $a$ in $\mathcal{A}$ under policy $\phi$ reach terminal states in a time whose mean is also less than $V(a) / \epsilon$. This holds for all $a$ and $\phi$.

We now prove that the technique is complete for finitely branching systems.

Definition 5. A probabilistic abstract reduction system $\mathcal{A}=(A, \rightarrow)$ is finitely branching if for all $a$, there is at most a finite number of distributions $\mu$ with $a \rightarrow \mu$. 
Theorem 3 (Completeness for finitely branching systems). If a finitely branching probabilistic abstract reduction system $\mathcal{A}=(A, \rightarrow)$ is + a.s. terminating then there exist some function $V: A \rightarrow \mathbb{R}$, with $\inf _{i \in A} V(i)>-\infty$, and some $\epsilon>0$, such that, for all states $a \in A$, for all $\mu$ with $a \rightarrow \mu$, the drift in a according to $\mu$ defined by

$$
\Delta_{\mu} V(a)=\sum_{i} \mu(i) V(i)-V(a)
$$

satisfies

$$
\Delta_{\mu} V(a) \leq-\epsilon .
$$

Proof. By hypothesis, for all states $a$, and policy $\phi$, we have $T[a, \phi]<+\infty$. When $h$ is a realizable history, and $\phi$ is a policy, we write $T[h, \phi]$ for the mean time before reaching $\perp$ after history $h$.

Note that for any policy $\phi$, when $h$ is a realizable non-terminal history, we have

$$
T[h, \phi]=1+\sum_{x \in A} \phi(h)(x) T[h x, \phi]
$$

If policy $\phi$ is Markovian, we have $T[h x, \phi]=T[x, \phi]$, and hence

$$
T[h, \phi]=1+\sum_{x \in A} \phi(h)(x) T[x, \phi] .
$$

The idea is to consider the "worst" strategy $\Phi$. This strategy can be built as follows: in any realizable non-terminal history $h=a_{0} \ldots a_{n}, \Phi$ maps $h$ to the distribution $\mu$ with $a_{n} \rightarrow \mu$ that maximizes $\sup _{\phi} \sum_{x \in A} \mu(x) T[h x, \phi]$.

Since to any strategy $\phi$ on can associate a strategy $\phi^{\prime}$ with

$$
T[h x, \phi]=T\left[x, \phi^{\prime}\right]
$$

(take $\phi^{\prime}\left(h^{\prime}\right)=\phi\left(h h^{\prime}\right)$ for any realizable non-terminal history $\left.h^{\prime}\right)$,

$$
\sup _{\phi} \sum_{x \in A} \mu(x) T[h x, \phi]=\sup _{\phi} \sum_{x \in A} \mu(x) T[x, \phi],
$$

and hence $\Phi$ is Markovian.

We claim that this is indeed the worst strategy, i.e.

$$
\sup _{\phi} T[h, \phi] \leq T[h, \Phi]
$$

for all realizable non-terminal histories $h$.

This follows from the following arguments: for any integer $i$, let $\Phi_{i}$ be the set of strategies that coincide with $\Phi$ on all histories of length less than $i$. Using repeatedly Equation 1, one gets for all integers $i$,

$$
\sup _{\phi} T[h, \phi] \leq \sup _{\phi \in \Phi_{i}} T[h, \phi]
$$


for all realizable non-terminal histories $h$ of length less than $i$.

Now, since $T[h, \Phi]$ is the limit of $\sup _{\phi \in \Phi_{i}} T[h, \phi]$ when $i$ goes to infinity, Equation 3 holds.

Now, in any non-terminal $a$, with $a \rightarrow \mu$,

$$
\begin{aligned}
\sum_{x \in A} \mu(x) T[x, \Phi] & \leq \sup _{\phi} \sum_{x \in A} \mu(x) T[x, \phi] \\
& \leq \sup _{\phi} \sum_{x \in A} \Phi(a)(x) T[x, \phi] \\
& \leq \sum_{x \in A} \Phi(a)(x) T[x, \Phi] .
\end{aligned}
$$

where first inequality comes from the fact that $\Phi$ is a particular strategy, the second from the definition of $\Phi(a)(x)$, and the third from the fact that the sup of a sum is always less that the sum of the sups.

We are done: indeed, if we take $V(a)=T[a, \Phi]$ for all states $a$, and $\epsilon=1$, we know that $V$ is non-negative, and for any $\mu$ with $a \rightarrow \mu$, we have

$$
\begin{aligned}
\Delta_{\mu} V(a) & =\sum_{x \in A} \mu(x) V(x)-V(a) \\
& =\sum_{x \in A} \mu(x) T[x, \Phi]-T[a, \Phi] \\
& =-1+\left(\sum_{x \in A} \mu(x) T[x, \Phi]-\sum_{x \in A} \Phi(a)(x) T[x, \Phi]\right) \\
& \leq-1
\end{aligned}
$$

where third equality comes from Equation 2, and last inequality from Equation 4 .

Remark 4. Note that the restriction to finitely branching systems in the previous theorem is mandatory: this can be seen as a consequence of Remark 3. Indeed, consider the counter-example after Proposition 1, considered as a probabilistic abstract reduction system. If there were a function $V$ and some $\epsilon>0$ as in the conclusion of previous theorem, adding a constant if necessary, and multiplying by $1 / \epsilon$ if necessary, we can assume $V$ non-negative, and $\epsilon=1$. Now, in any non-terminal state $a$ with $a \rightarrow \mu$, since we should have $\Delta_{\mu} V(a) \leq-1$, and since $\mu$ is a Dirac that is 0 except on some point $x$ where it has value 1 , we must have for that $x, V(x) \leq V(a)-1$. Now, if $k=V(1,1)$, consider the strategy going from $(1,1)$ to $(0, k),(0, k-1), \ldots,(0,0) . V$ must decrease of at least 1 at each transition. That leads to a contradiction, since starting from $k$, one can not do it $k+1$ times keeping $V$ non-negative.

\section{Probabilistic Rewrite Systems}

We are now introducing the notion of probabilistic rewrite system. Our motivation is to get something that covers classical (i.e. non-probabilistic) rewrite systems, and also Markov chains over finite spaces. Doing so, we can claim that all examples that have been modeled in literature using finite Markov chains (for e.g. in model-checking contexts [14]) can be modeled in this framework.

Definition 6 (Probabilistic Rewrite system). Given a signature $\Sigma$ and a set of variables $X$, the set of terms over $\Sigma$ and $X$ is denoted by $T(\Sigma, X)$.

A probabilistic rewrite rule is an element of $T(\Sigma, X) \times \operatorname{Dist}(T(\Sigma, X))$. A probabilistic rewrite system is a finite set $\mathcal{R}$ of probabilistic rewrite rules. 
To a probabilistic rewrite system is associated a probabilistic abstract reduction system $\left(T(\Sigma, X), \rightarrow_{\mathcal{R}}\right)$ over the set of terms $T(\Sigma, X)$ where $\rightarrow_{\mathcal{R}}$ is defined as follows: When $t \in T(\Sigma, X)$ is a term, let $\operatorname{Pos}(t)$ be the set of its positions. For $\rho \in \operatorname{Pos}(t)$, let $\left.t\right|_{\rho}$ be the subterm of $t$ at position $\rho$, and let $t[s]_{\rho}$ denote the replacement of the subterm at position $\rho$ in $t$ by $s$. The set of all substitutions is denoted by $S u b$.

Definition 7 (Reduction relation). To a probabilistic rewrite system $\mathcal{R}$ is associated the following PARS $(T(\Sigma, X), \rightarrow)$ over terms:

$$
t \rightarrow_{\mathcal{R}} \mu
$$

iff there is a rule $(g, M) \in \mathcal{R}$, some position $p \in \operatorname{Pos}(t)$, some substitution $\sigma \in S u b$, such that $\left.t\right|_{p}=\sigma(g)$, and, for all $t^{\prime}$,

$$
\mu\left(t^{\prime}\right)=\sum_{t^{\prime}=t[\sigma(d)]_{p}} M(d) .
$$

For example, a probabilistic rewrite rule can be $f(x, y) \mapsto g(a): 1 / 2 \mid y: 1 / 2$, where $g(a): 1 / 2 \mid y: 1 / 2$ denotes the distribution with value $1 / 2$ on $g(a)$ and value $1 / 2$ on $y$. Then $f(b, c)$ rewrites to $g(a)$ with probability $1 / 2$, and to $c$ with probability $1 / 2$. Now, $f(b, g(a))$ rewrites to $g(a)$ with probability 1 .

\section{Termination of a Probabilistic Rewrite System}

We now provide an equivalent of the result that says that a rewrite system is terminating iff there is a reduction order monotone on each rewrite rule $[1,13]$.

Theorem 4. A probabilistic rewrite system $\mathcal{R}$ is positively almost surely terminating if and only if there exists some function $V: T(\Sigma, X) \rightarrow \mathbb{R}$, with $\inf _{i \in A} V(i)>-\infty$, and some $\epsilon>0$, such that

1. "the drift of each rule is less than $-\epsilon$ ": for each probabilistic rewrite rule $g \rightarrow M \in \mathcal{R}$, the drift

$$
\Delta_{M} V(g)=\sum_{d} M(d)(V(d)-V(g))
$$

satisfies

$$
\Delta_{M} V(g) \leq-\epsilon .
$$

2. "drift being less than $-\epsilon$ is preserved by substitutions":

for each term $s \in T(\Sigma, X)$, for all $\mu$ with $s \rightarrow \mu$, for all substitutions $\sigma \in$ $S u b$, if $\Delta_{\mu} V(s) \leq-\epsilon$ then the drift

$$
\Delta_{\sigma(\mu)} V(\sigma(s))=\sum_{s^{\prime}} \mu\left(s^{\prime}\right)\left(V\left(\sigma\left(s^{\prime}\right)\right)-V(\sigma(s))\right)
$$

satisfies

$$
\Delta_{\sigma(\mu)} V(\sigma(s)) \leq-\epsilon
$$


3. "drift being less than $-\epsilon$ is preserved by contexts": for each term $s_{1}, \ldots, s_{n}, s \in$ $T(\Sigma, X)$, for all $\mu$ with $s \rightarrow \mu$, for all function symbols $f$, if $\Delta_{\mu} V(s) \leq-\epsilon$, then the drift

$$
\begin{aligned}
\Delta_{f\left(s_{1}, \ldots, \mu, \ldots, s_{n}\right)} V\left(f\left(s_{1}, \ldots, s, \ldots, s_{n}\right)\right)= & \sum_{s^{\prime}} \mu\left(s^{\prime}\right)\left(V\left(f\left(s_{1}, \ldots, s^{\prime}, \ldots, s_{n}\right)\right)\right. \\
& \left.-V\left(f\left(s_{1}, \ldots, s, \ldots, s_{n}\right)\right)\right)
\end{aligned}
$$

satisfies

$$
\Delta_{f\left(s_{1}, \ldots, \mu, \ldots, s_{n}\right)} V\left(f\left(s_{1}, \ldots, s, \ldots, s_{n}\right)\right) \leq-\epsilon .
$$

Proof. If $\mathcal{R}$ is positively almost surely terminating, then by Theorem 3 , there exists some function $V: T(\Sigma, X) \rightarrow \mathbb{R}$, with $\inf _{i \in A} V(i)>-\infty$, and some $\epsilon>0$, such that, for all states $a \in T(\Sigma, X)$, for all $\mu$ with $a \rightarrow \mu, \Delta_{\mu} V(a) \leq-\epsilon$.

In particular, for $a=g$, we have $a \rightarrow \mu$, where $\mu\left(t^{\prime}\right)=M\left(t^{\prime}\right)$, and hence

$$
\begin{aligned}
\Delta_{\mu} V(a) & =\sum_{t^{\prime}} \mu\left(t^{\prime}\right) V\left(t^{\prime}\right)-V(a) \\
& =\sum_{d} M(d)(V(\sigma(d))-V(a)) \\
& =\Delta_{M} V(g) \\
& \leq-\epsilon .
\end{aligned}
$$

Now, when $s \rightarrow \mu^{\prime}$, for $a=\sigma(s)$, we have $a \rightarrow \mu$, where $\mu\left(\sigma\left(s^{\prime}\right)\right)=\mu^{\prime}\left(s^{\prime}\right)$, and hence

$$
\begin{aligned}
\Delta_{\mu} V(a) & =\sum_{t^{\prime}} \mu\left(t^{\prime}\right) V\left(t^{\prime}\right)-V(a) \\
& =\sum_{t^{\prime}=\sigma\left(s^{\prime}\right)} \mu^{\prime}\left(s^{\prime}\right) V\left(\sigma\left(s^{\prime}\right)\right)-V(a) \\
& =\Delta_{\sigma\left(\mu^{\prime}\right)} V(\sigma(s)) \\
& \leq-\epsilon .
\end{aligned}
$$

In a same way, when $s \rightarrow \mu^{\prime}$, for $a=f\left(s_{1}, \ldots, s, \ldots, s_{n}\right)$, we have $a \rightarrow \mu$ where $\mu\left(f\left(s_{1}, \ldots, s^{\prime}, \ldots, s_{n}\right)\right)=\mu^{\prime}\left(s^{\prime}\right)$, and hence

$$
\begin{aligned}
\Delta_{\mu} V(a) & =\sum_{t^{\prime}} \mu\left(t^{\prime}\right) V\left(t^{\prime}\right)-V(a) \\
& =\sum_{s^{\prime}} \mu^{\prime}\left(s^{\prime}\right) V\left(f\left(s_{1}, \ldots, s^{\prime}, \ldots, s_{n}\right)\right)-V\left(f\left(s_{1}, \ldots, s, \ldots, s_{n}\right)\right. \\
& =\Delta_{f\left(s_{1}, \ldots, \mu^{\prime}, \ldots, s_{n}\right)} V\left(f\left(s_{1}, \ldots, s, \ldots, s_{n}\right)\right) \\
& \leq-\epsilon .
\end{aligned}
$$

This proves that conditions 1,2 and 3 are necessary.

Conversely, assume that conditions 1,2 and 3, hold. We have $t \rightarrow \mu$ iff there is a rule $(g, M) \in \mathcal{R}$, some position $p \in \operatorname{Pos}(t)$, some substitution $\sigma \in S u b$, such that $\left.t\right|_{p}=\sigma(g)$, and, for all $t^{\prime}, \mu\left(t^{\prime}\right)=\sum_{t^{\prime}=t[\sigma(d)]_{p}} M(d)$.

Since a derivation $t \rightarrow \mu$ is necessarily via some rule $(g, M)$, from Theorem 2 , we only need to prove that for all rules $(g, M)$ and term $t$, if $t \rightarrow \mu$ via $(g, M)$ then $\Delta \mu V(t) \leq-\epsilon$.

This is proved by induction on the length of $p$. If $p$ is of length 0 , then $t=\sigma(g)$. By condition 1, we know that $\Delta_{M} V(g) \leq-\epsilon$. By condition 2, since $g \rightarrow M$, and $\Delta_{M} V(g) \leq-\epsilon$, we have $\Delta_{\mu} V(t)=\Delta_{\sigma(M)} V(\sigma(g)) \leq-\epsilon$, where the equality is established as in the third paragraph above.

If $p=p_{1} p_{2} \ldots p_{k}$ is of length $k>0$, then $t$ can be written as $f\left(s_{1}, \ldots, s, \ldots, s_{n}\right)$ and $s \rightarrow \mu^{\prime}$ via $(g, M)$. By induction hypothesis, $\Delta_{\mu^{\prime}} V(s) \leq-\epsilon$. By condition $3, \Delta_{\mu} V(t)=\Delta_{f\left(s_{1}, \ldots, \mu^{\prime}, \ldots, s_{n}\right)} V\left(f\left(s_{1}, \ldots, s, \ldots, s_{n}\right)\right) \leq-\epsilon$, where the equality is established as in the fourth paragraph above. 
Sufficient conditions for 1, 2 and 3 can be established. Indeed:

Definition 8 (Context preservation of a function). A function $V: T(\Sigma, X) \rightarrow$ $\mathbb{R}$ is context preserving if for all $t, t^{\prime}, s_{1}, \ldots, s_{n}$ and function symbol $f$,

$$
V\left(f\left(s_{1}, \ldots, t, \ldots, s_{n}\right)\right)-V\left(f\left(s_{1}, \ldots, t^{\prime}, \ldots, s_{n}\right)\right)=V(t)-V\left(t^{\prime}\right) .
$$

Definition 9 (Substitution decrease on a rule). A function $V: T(\Sigma, X) \rightarrow$ $\mathbb{R}$ is substitution decreasing on a probabilistic rewrite rule $(g, M)$, if for all substitution $\sigma \in S u b$, if we denote

$$
\Delta_{\sigma(M)} V(\sigma(g))=\sum_{d} M(d)(V(\sigma(d))-V(\sigma(g)))
$$

and $\Delta_{M} V(g)=\sum_{d} M(d)(V(d)-V(g))$ as before, we have

$$
\Delta_{\sigma(M)} V(\sigma(g)) \leq \Delta_{M} V(g) .
$$

Theorem 5. A probabilistic rewrite system $\mathcal{R}$ is positively almost surely terminating if there exists some function $V: T(\Sigma, X) \rightarrow \mathbb{R}$, with $\inf _{i \in A} V(i)>-\infty$, and some $\epsilon>0$, such that the drift of each rule is less or equal to $-\epsilon, V$ is context preserving, and $V$ is substitution decreasing on every rule.

Proof. Condition 1 holds by hypothesis.

Since $V$ is context preserving, for all $f, s, s_{1}, \ldots, s_{n}$ and $\mu$, we have

$$
\Delta_{f\left(s_{1}, \ldots, \mu, \ldots, s_{n}\right)} V\left(f\left(s_{1}, \ldots, s, \ldots, s_{n}\right)\right)=\Delta_{\mu} V(s)
$$

and so, condition 3 holds.

Now, given conditions 1 and 3 , the proof of indirect sense of Theorem 4, only require that $\Delta_{\sigma(M)} V(\sigma(g))=\sum_{d} M(d)(V(\sigma(d))-V(\sigma(g))) \leq-\epsilon$ for each probabilistic rule $(g, M)$ and substitution $\sigma$. Now, this holds, since $V$ is substitution decreasing and so $\Delta_{\sigma(M)} V(\sigma(g)) \leq \Delta_{M} V(g) \leq-\epsilon$, by condition 1 .

We are now discussing some examples:

Example 1. The probabilistic rewrite system restricted to the unique rule

$$
a \rightarrow a: 1 / 2 \mid b: 1 / 2
$$

is + a.s. terminating. Indeed, consider $V(a)=10, V(b)=2$, and observe that $1 / 2 \times 10+1 / 2 \times 2-10<0$.

Example 2. The probabilistic rewrite system

$$
\begin{aligned}
& f(x) \rightarrow x: p_{1} \mid f(f(x)): 1-p_{1} \\
& f(x) \rightarrow x: p_{2} \mid f(f(x)): 1-p_{2}
\end{aligned}
$$

is + a.s. terminating if $p_{1}>1 / 2$ and $p_{2}>1 / 2$. Indeed, consider $V$ that returns the size of a term. $V$ is easily shown context preserving. $V$ is also easily shown substitution decreasing on both rules. Now the drift of each rule is given by $-1 \times p_{i}+1 \times\left(1-p_{i}\right)=1-2 p_{i} \leq \min \left(1-2 p_{1}, 1-2 p_{2}\right)<0$. 
Example 3. The probabilistic rewrite system

$$
\begin{array}{ll}
f(x) & \rightarrow f(f(x)): p_{1_{1}}\left|g(f(x)): p_{1_{2}}\right| x: p_{1_{3}} \\
f(h(f(x), x)) & \rightarrow h(g(f(f(x))), f(x)): p_{2_{1}}\left|g(f(x)): p_{2_{2}}\right| f(g(f(f(x)))): p_{2_{3}}
\end{array}
$$

It is + a.s. terminating if $p_{1_{1}}+p_{1_{3}}<p_{1_{3}}$ and $p_{2_{1}}<p_{2_{2}}$. Indeed, consider same function $V$, which is clearly context preserving. An easy computation shows that the drift of the first rule $\left(g_{1}, M_{1}\right)$ is $\Delta_{M_{1}} V\left(g_{1}\right)=p_{1_{1}}+p_{1_{2}}-p_{1_{3}}$. For the second rule $\left(g_{2}, M_{2}\right)$, we have $\Delta_{M_{2}} V\left(g_{2}\right)=2 p_{2_{1}}-2 p_{2_{2}}$. Hence, both are negative. Now $V$ is substitution decreasing on both rules: given some substitution $\sigma \in S u b$, if we denote $n=V(\sigma(x))$, some easy computations show that we have $\Delta_{\sigma\left(M_{1}\right)} V\left(\sigma\left(g_{1}\right)\right)=p_{1_{1}}+p_{1_{2}}-p_{1_{3}}=\Delta_{M_{1}} V\left(g_{1}\right)$ and $\Delta_{\sigma\left(M_{2}\right)} V\left(\sigma\left(g_{2}\right)\right)=$ $\left(p_{2_{1}}-1\right) n+2 p_{2_{1}}-p_{2_{2}}+p_{2_{3}} \leq 2 p_{2_{1}}-2 p_{2_{2}}=\Delta_{M_{2}} V\left(g_{2}\right)$ since $n \geq 1$ and $\left(p_{2_{1}}-1\right)=-p_{2_{2}}-p_{2_{3}}<0$.

\section{Conclusion and perspective}

In this paper we presented non-deterministic probabilistic abstract reduction systems, probabilistic rewrite systems, and we gave necessary and sufficient conditions for proving positive and almost sure termination of these systems. We also provided tractable sufficient conditions and application examples.

We believe that our notion of probabilistic rewrite system is very powerful since it covers all systems that can be encoded by classical rewrite systems and finite Markov chains. In particular, we already explored it to model a telecommunication protocol.

Next step include understanding whether there could be valid and interesting results generalizing techniques based on polynomial orders, or even on semantical methods.

\section{Acknowledgment}

We would like to thanks Thierry Heuillard for pointing out the fact that for protocols, and in almost all other contexts, only positive almost sure termination is interesting. His remarks and suggestions motivated a great part of this work. We would also like to thanks Claude Kirchner for many very fruitful discussions on this work.

\section{References}

1. Franz Baader and Tobias Nipkow. Term Rewriting and All That. Cambridge University Press, 1998.

2. Gianfranco Balbo. Introduction to Stochastic Petri nets. Lecture Notes in Computer Science, 2090:84, Springer-Verlag, 2001.

3. Olivier Bournez and Mathieu Hoyrup. Rewriting Logic and Probabilities. In Robert Nieuwenhuis, editor, RTA 2003, volume 2706 of Lecture Notes in Computer Science, pages 61-75. Springer-Verlag, 2003. 
4. Olivier Bournez and Claude Kirchner. Probabilistic Rewrite Strategies: Applications to ELAN. In Sophie Tison, editor, RTA 2002, volume 2378 of Lecture Notes in Computer Science, pages 252-266. Springer-Verlag, 2002.

5. Pierre Brémaud. Markov Chains, Gibbs Fields, Monte Carlo Simulation, and Queues. Springer-Verlag, New York, 2001.

6. L. de Alfaro. Formal Verification of Probabilistic Systems. PhD thesis, Stanford University, 1997.

7. W. Feller. An Introduction to Probability Theory and its Applications, volume 1. Wiley, 1968.

8. F. G. Foster. On the Stochastic Matrices Associated with Certain Queuing Processes. The Annals of Mathematical Statistics, 24:355-360, 1953.

9. Thom Frühwirth, Alexandra Di Pierro, and Herbert Wiklicky. Toward Probabilistic Constraint Handling Rules. In Slim Abdennadher and Thom Frühwirth, editors, RCoRP'01, 2001.

10. G. Grimmett. Probability Theory. Cambridge University Press, 1993.

11. H. Hansson. Time and Probability in Formal Design of Distributed Systems. Series in Real-Time Safety Critical Systems. Elsevier, 1994.

12. Claire Jones. Probabilistic Non-determinism. PhD thesis, University of Edinburgh, 1990.

13. Jan Willem Klop. Term Rewriting Systems. In S. Abramsky, D. M. Gabbay, and T. S. E. Maibaum, editors, Handbook of Logic in Computer Science, volume 2, chapter 1, pages 1-117. Oxford University Press, Oxford, 1992.

14. Marta Kwiatkowska, Gethin Norman, and David Parker. PRISM: Probabilistic Symbolic Model Checker. Lecture Notes in Computer Science, 2324:200, SoringerVerlag 2002.

15. N. Lynch. Distributed Algorithms. Morgan Kaufmann Publishers, Inc, 1997.

16. Narciso Martí-Oliet and José Meseguer. Rewriting Logic: Roadmap and Bibliography. Theoretical Computer Science, 285(2):121-154, 2002.

17. J. Meseguer. Conditional Rewriting Logic as a Unified Model of Concurrency. Theoretical Computer Science, 96(1):73-155, 1992.

18. Kumar Nirman, Koushik Sen, Jose Meseguer, and Gul Agha. A Rewriting Based Model for Probabilistic Distributed Object Systems. In FMOODS'03, volume 2884 of Lecture Notes in Computer Science, pages 32-46, Springer-Verlag 2003.

19. Panangaden. Does Combining Probability and Non-Determinism Makes Sense? Bulletin of the EATCS, 2001.

20. M.L. Puternam. Markov Decision Processes - Discrete Stochastic Dynamic Programming. Wiley Series in Probability and Mathematical Statistics. John Wiley \& Sons, 1994.

21. W. Rudin. Real and Complex Analysis, 3rd edition. McGraw Hills, USA, 1987.

22. William H. Sanders and John F. Meyer. Stochastic Activity Networks: Formal Definitions and Concepts. Lecture Notes in Computer Science, 2090:315, SpringerVerlag 2001.

23. M. Schneider. Self-stabilization. ACM Computing Surveys, 25:45-67, 1993.

24. R. Segala and N. Lynch. Probabilistic Simulations for Probabilistic Processes. Lecture Notes in Computer Science, 836:481, Sringer-Verlag 1994.

25. Rob van Glabbeek, Scott A. Smolka, Bernhard Steffen, and Chris M. N. Tofts. Reactive, Generative, and Stratified Models of Probabilistic Processes. In LICS'90, pages 130-141, 1990. IEEE Computer Society Press.

26. M. Y. Vardi. Automatic Verification of Probabilistic Concurrent Finite-State Programs. In $F O C S^{\prime} 85$, pages 327-338, 1985. 